\title{
Characterizing closure-phase measurements at IOTA
}

Sam Ragland, Wesley Arthur Traub, Jean-Philippe Berger, Rafael Millan-Gabet, John D. Monnier, et al.

Sam Ragland, Wesley Arthur Traub, Jean-Philippe Berger, Rafael MillanGabet, John D. Monnier, Ettore Pedretti, F. Peter Schloerb, Nathaniel P. Carleton, Pierre Haguenauer, Pierre Y. Kern, Pierre R. Labeye, Marc G. Lacasse, Fabien Malbet, Karine Rousselet-Perraut, "Characterizing closurephase measurements at IOTA," Proc. SPIE 5491, New Frontiers in Stellar Interferometry, (20 October 2004); doi: 10.1117/12.552447

Event: SPIE Astronomical Telescopes + Instrumentation, 2004, Glasgow, United Kingdom 


\title{
Characterizing Closure-phase Measurements at IOTA
}

\author{
Ragland, S. ${ }^{1,2,3}$, Traub, W. ${ }^{1}$, Berger, J.-P. ${ }^{4}$, Millan-Gabet, R. ${ }^{5}$, Monnier, J. D. ${ }^{6}$, Pedretti, E. ${ }^{6}$, \\ Schloerb, F. P. ${ }^{7}$, Carleton, N. P. ${ }^{1}$, Haguenauer, P. ${ }^{8}$, Kern, P. ${ }^{4}$, Labeye, P. , Lacasse, M. G. ${ }^{1}$, Malbet, \\ F. ${ }^{4}$, Perraut, K. ${ }^{4}$
}

\author{
${ }^{1}$ Harvard-Smithsonian Center for Astrophysics, Cambridge, MA 02138 \\ ${ }^{2}$ Present address: CARA, 65-1120 Mamalahoa Hwy, Kamuela, Hi 96743 \\ ${ }^{3}$ sragland@keck.hawaii.edu \\ ${ }^{4}$ Laboratoire d'Astrophysique de Grenoble, F-38400 Saint Martin d'Heres, France \\ ${ }^{5}$ Michelson Science Center, California Institute of Technology, Pasadena, CA 91125 \\ ${ }^{6}$ University of Michigan at Ann Arbor, Ann Arbor, MI 48109-1090 \\ ${ }^{7}$ University of Mass. at Amherst, Department of Astronomy, Amherst, MA 01003-9305 \\ ${ }^{8}$ ALCATEL Space Industries, 100 boulevard du Midi, BP99, 06322 Cannes, France \\ ${ }^{9}$ LETI, CEA-Grenoble, 17 rue des Martyrs, 38054 Grenoble CEDEX 9, France
}

\begin{abstract}
We are working towards imaging the surfaces and circumstellar envelopes of Mira stars in the near-infrared, using the IOTA interferometer and the IONIC integrated-optics 3-beam combiner. In order to study atmospheric structures of these stars, we installed 3 narrow-band filters that subdivide H-band into 3 roughly equal-width sub-bands - a central one for continuum, and 2 adjacent ones to sample Mira star's (mostly water) absorption-bands. We present here our characterization of the IOTA 3-Telescope interferometer for closure-phase measurements with broad and narrow-band filters in the $\mathrm{H}$ atmospheric window. This includes characterizing the stability, chromaticity, and polarization effects of the present IOTA optics with the IONIC beam-combiner, and characterizing the accuracy of our closure phase measurements.
\end{abstract}

Keywords: optical interferometry, closure phase technique, spatial filtering, integrated optics, imaging

\section{INTRODUCTION}

During the commissioning phase of the IOTA 3-telescope interferometer and the IONIC integrated optics 3-beam combiner, we initiated a shared-risk science program of imaging evolved stars in the near-infrared wavelengths. In order to characterize the IOTA-IONIC for the closure phase and visibility amplitude measurements, we took engineering data during those science observing runs. We investigate issues such as stability, chromatism and polarization of the instrument, and the role of instrumental settings on closure phase estimation. The results are briefly discussed here and more detailed version will be presented elsewhere.

\section{INSTRUMENTATION}

The Infrared-Optical Telescope Array (IOTA) is a long baseline interferometer, with demonstrated closure-phase capability, operating at visible and near-infrared wavelengths. The 3 IOTA telescopes each have a diameter of $0.45 \mathrm{~m}$, and a minimum-to-maximum baseline range of 5-38 $\mathrm{m}$. The telescopes are movable among 17 stations, along 2 linear arms, within this range. IOTA achieved first fringes with two telescopes in December 1993, and more recently, IOTA has been upgraded with the addition of a third telescope and a new control system for the interferometer (Traub et al. 2004). An aerial view of the IOTA 3-telescope interferometer is shown in Figure 1 (left).

Presently, the IOTA 3-telescope array is equipped with an integrated-optics-based beam-combiner operating in the $\mathrm{H}$ band (Berger et al., 2003). A photograph of the IONIC chip mounted on a XYZ stage is shown in Figure 1 (right). The optical layout of the IOTA-IONIC instrument is shown in Figure 2. The beams from 3 telescopes are fed to a tiny 
integrated-optics chip using single-mode optical fibers. Beam combination takes place inside the integrated-optics chip and the 6 interferometric outputs are re-imaged onto 6 pixels of a PICNIC camera. Thus, the IOTA interferometer has three advanced capabilities namely, integrated-optics-based beam combiner, spatial filtering of the wavefront, and measuring closure-phase quantity.
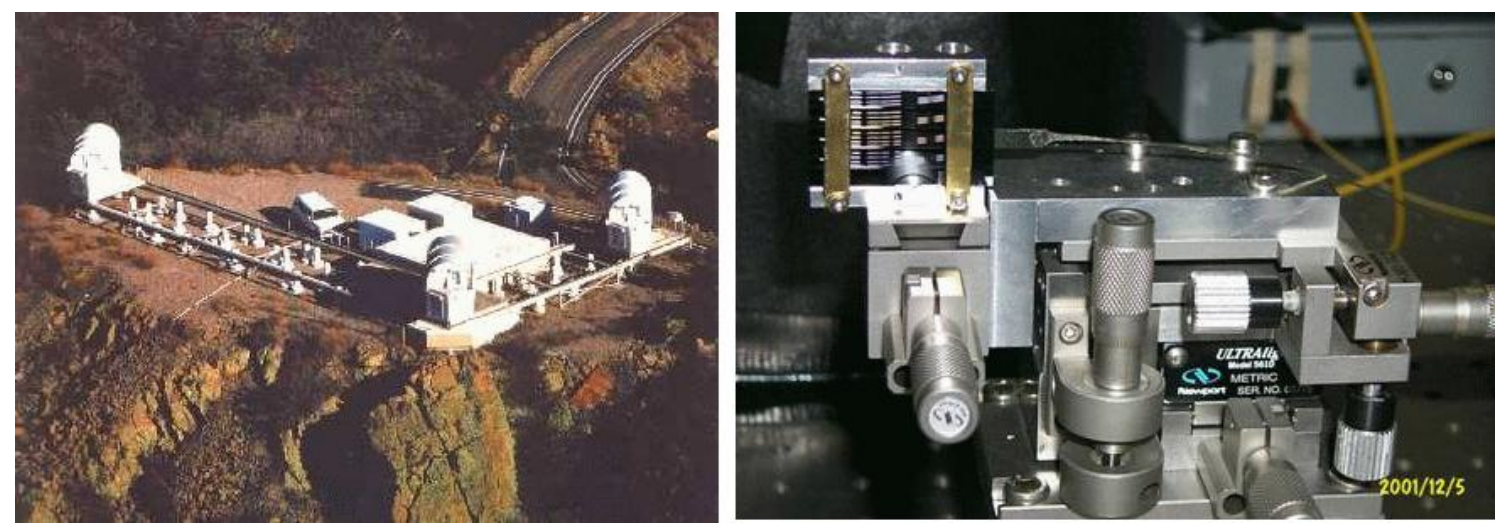

Figure 1. Left: The Infrared-Optical Telescope Array (IOTA). Right: The IONIC beam combiner at the IOTA

The presently existing integrated-optics-based 3-beam combiner at IOTA is supplied by our collaborators at Grenoble. Our collaboration with Grenoble is a long-term one, under which two IO components have been installed at IOTA (a 2-beam device in 2000, and a 3-beam device in 2001), and a third one in November 2002 (the present one, an improved 3-beam device). The observations presented here were taken with these 3-beam devices during seven observing runs spanning a period of about one-and-half years.

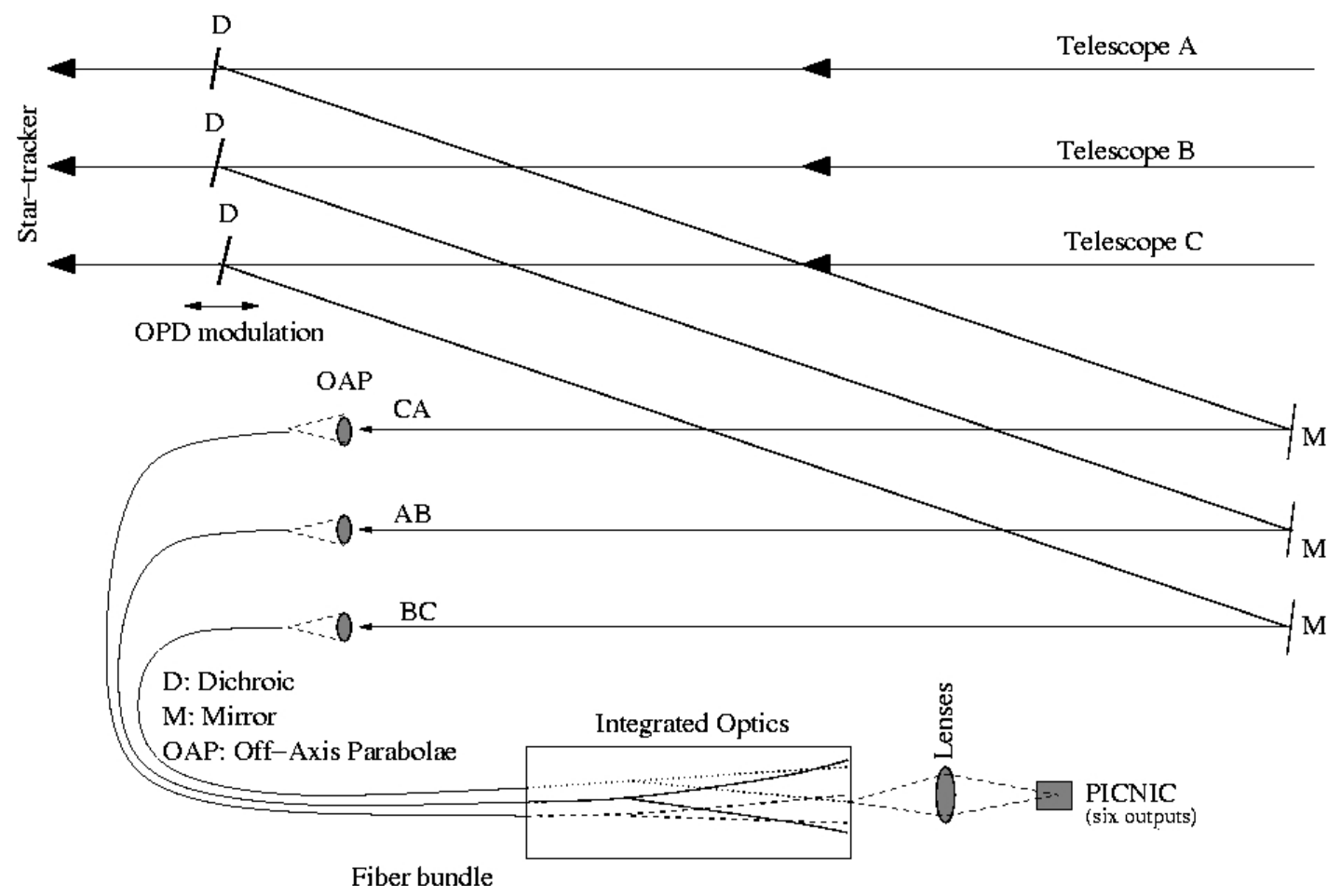

Figure 2. Optical layout of the IOTA-IONIC instrument 


\section{OBSERVATIONS}

The engineering data for the characterization presented here were taken during the commissioning phase of the IOTA 3-telescope interferometer during 2002-2003 as a part of the on going science program on AGB stars in the H-band $(1.65 \mu \mathrm{m})$ atmospheric window. Observations were taken either with a standard $\mathrm{H}$ band filter $(\lambda=1.65 \mu \mathrm{m}, \Delta \lambda=0.3$ $\mu \mathrm{m})$ or with three narrow-band filters. The central wavelengths of these filters are 1.50, 1.64 and $1.78 \mu \mathrm{m}$ with FWHM of $0.09,0.1$ and $0.1 \mu \mathrm{m}$ respectively. These targets were observed simultaneously in three baselines forming closed triangles. The fringe tracking is performed by algorithms presented in Pedretti et al. (2004). We measured 3 visibility amplitudes and 1 closure phase for each star. In addition, the earth's rotation enables measurements at slightly different projected baselines (and hence different closed triangles) when observations are made at different hour angles. We have adopted baseline bootstrapping at the IOTA whereby fringes are tracked in two short baselines, while science data are recorded in all three baselines simultaneously, enabling low visibility closure-phase measurements on the third baseline. IOTA's maximum baseline of $38 \mathrm{~m}$ yields an angular resolution of about 4 mas at $1.65 \mu \mathrm{m}$.

The recorded interferograms were reduced with an IDL code developed by one of us (S. Ragland). Our data reduction procedure accounts for low frequency photometric fluctuations of telescope beams that are subject to interference, and thereby enables accurate visibility and closure phase estimation from our spatially filtered interferograms. We have worked out a scheme to carry out these corrections from science data itself knowing the coupling efficiency of the beam-combiner (Ragland et al., 2004). Simultaneous interferograms taken with the three-telescope configuration are shown in Figure 3. For clarity, the complimentary outputs are not shown here. The corrected interferograms after low frequency photometric fluctuations are taken into account are also shown in the same figure.
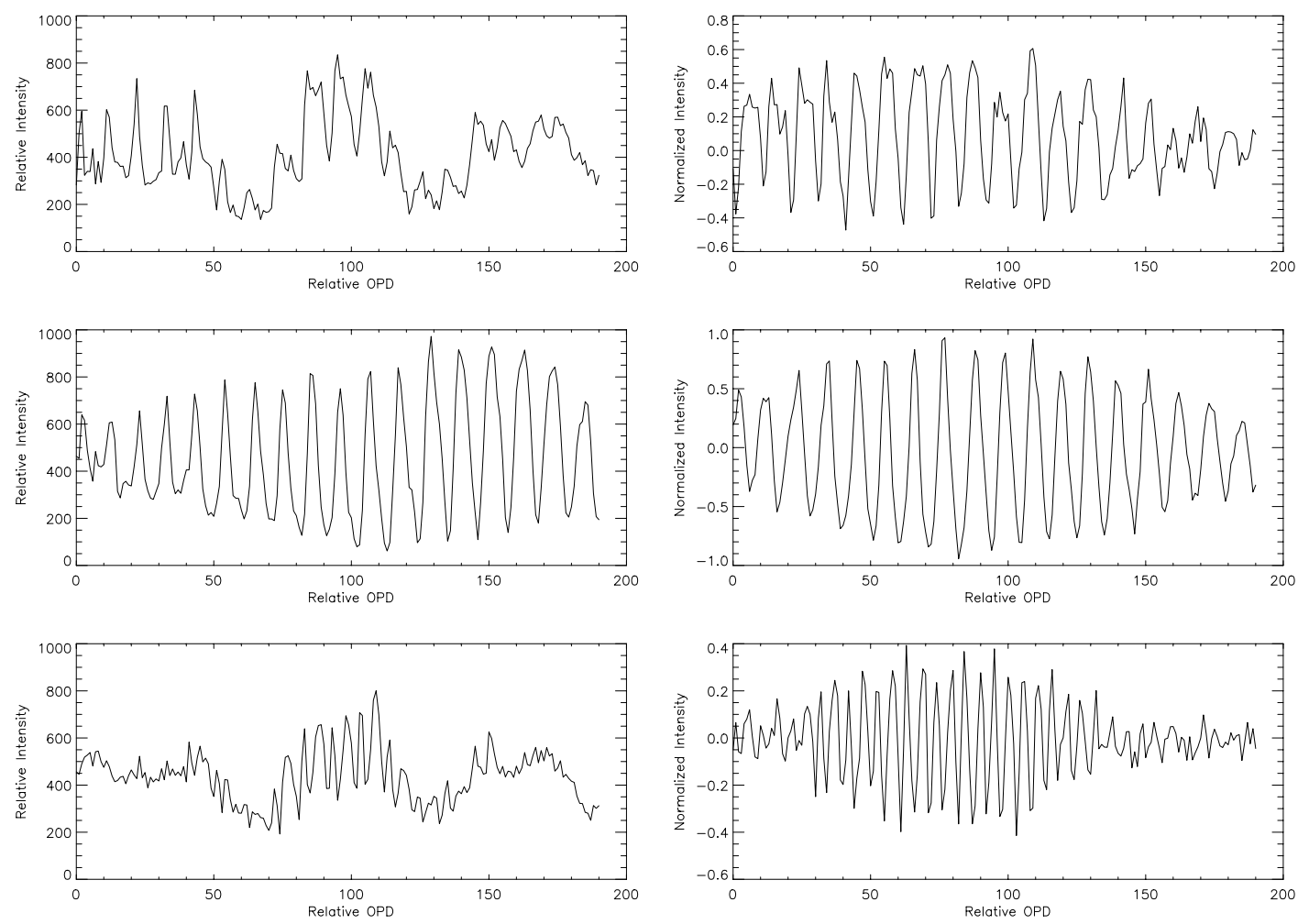

Figure 3. Left: A single scan data of R Ser taken on $13^{\text {th }}$ May 2003, showing simultaneous fringes from all three baselines of the three-telescope configuration at IOTA (for clarity, the complimentary outputs are not shown here). Right: The corrected interferograms after low frequency photometric fluctuations are taken into account. 


\section{CHARACTERIZATION}

Issues such as stability, chromatism and polarization effects of the IOTA-IONIC instrument are discussed here briefly. In addition, the dependence of the instrumental closure phase on the readout pattern of the PICNIC detector and the sensitivity of the instrument are also discussed briefly.

The formal error in our closure phase estimations for bright unresolved stars is better than about 0.5 degrees. Taking into account the systematic errors, the typical accuracy of our closure phase measurements is about a degree for bright science targets.

\subsection{Stability of the IOTA-IONIC}

The IOTA-IONIC enables extremely stable day-to-day and run-to-run closure phase measurements of about a degree with the narrowband filters in the $\mathrm{H}$ band (Figure 4). The standard $\mathrm{H}$ filter measurements show stability at the level of 2-3 degrees. The stability of visibility amplitude measurements during our Dec. 2003 run is shown in Figure 5.
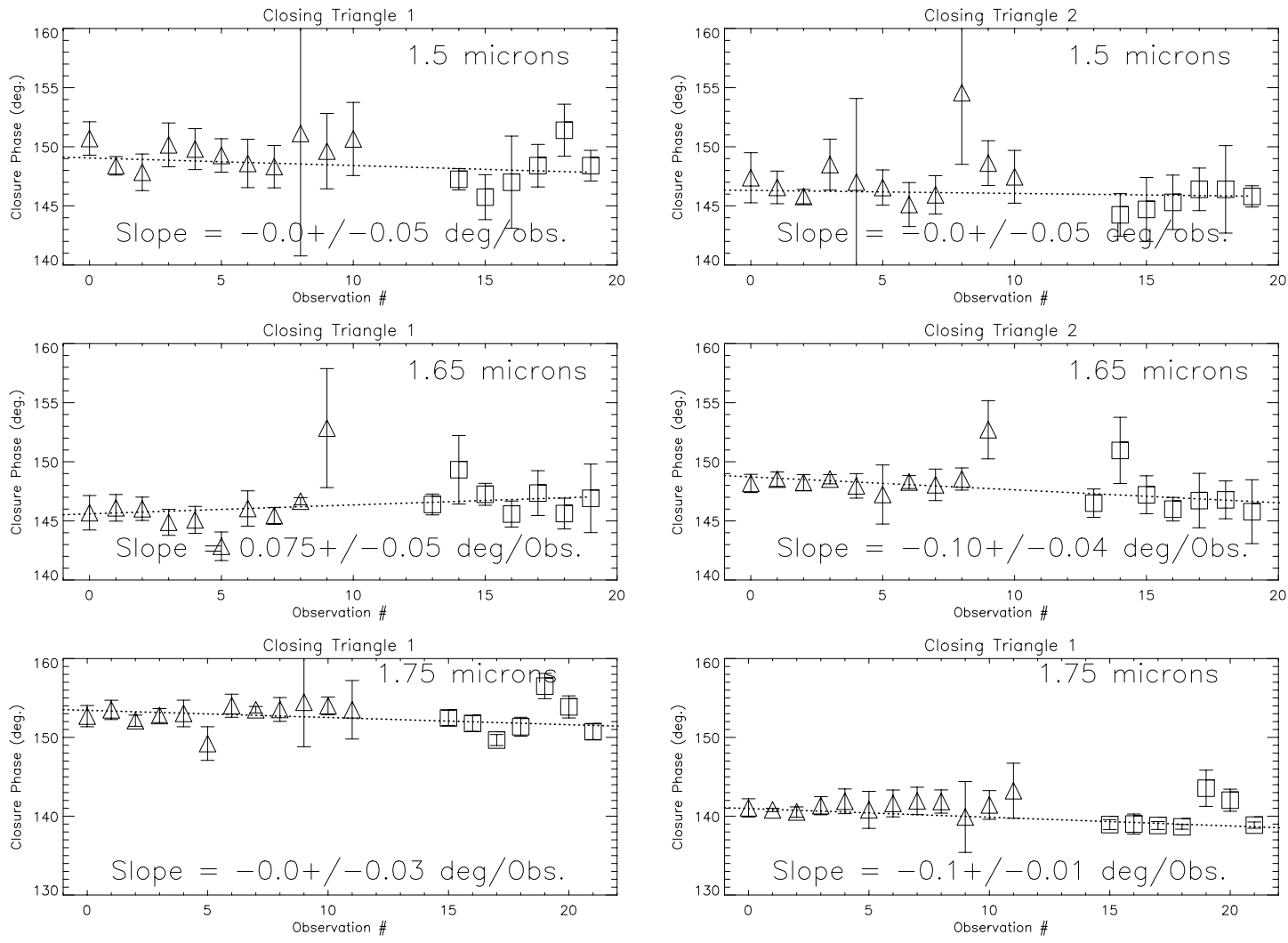

Figure 4. Instrumental closure phase in Mar 2003 (triangles) \& May 2003 (squares) are shown here. Run-to-run stability of IOTA-IONIC for closure phase measurements is about 1 degree. 

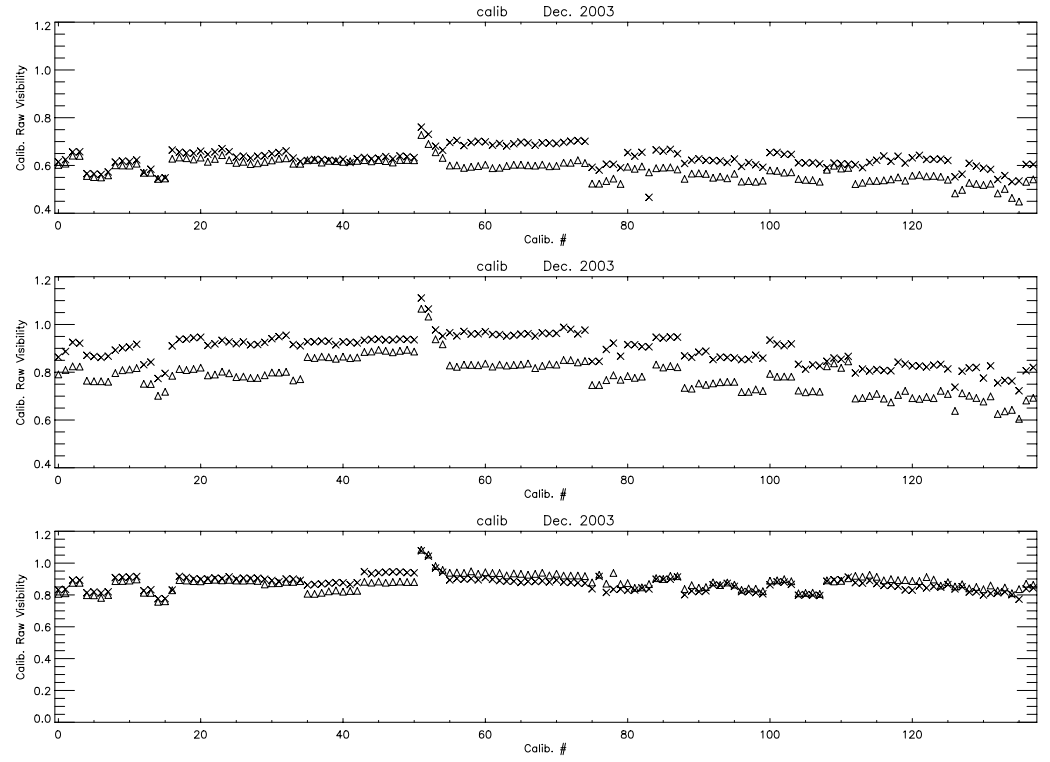

Figure 5. Stability of our visibility amplitude is shown using calibrators observed during Dec. 2003. Each panel consists of two complimentary outputs corresponds to each baseline.

\subsection{Chromatism of the IOTA-IONIC}

We find that the instrumental closure phase is almost independent of the spectral type of the observed star within the typical observational errors. These results are shown in Figure 6.
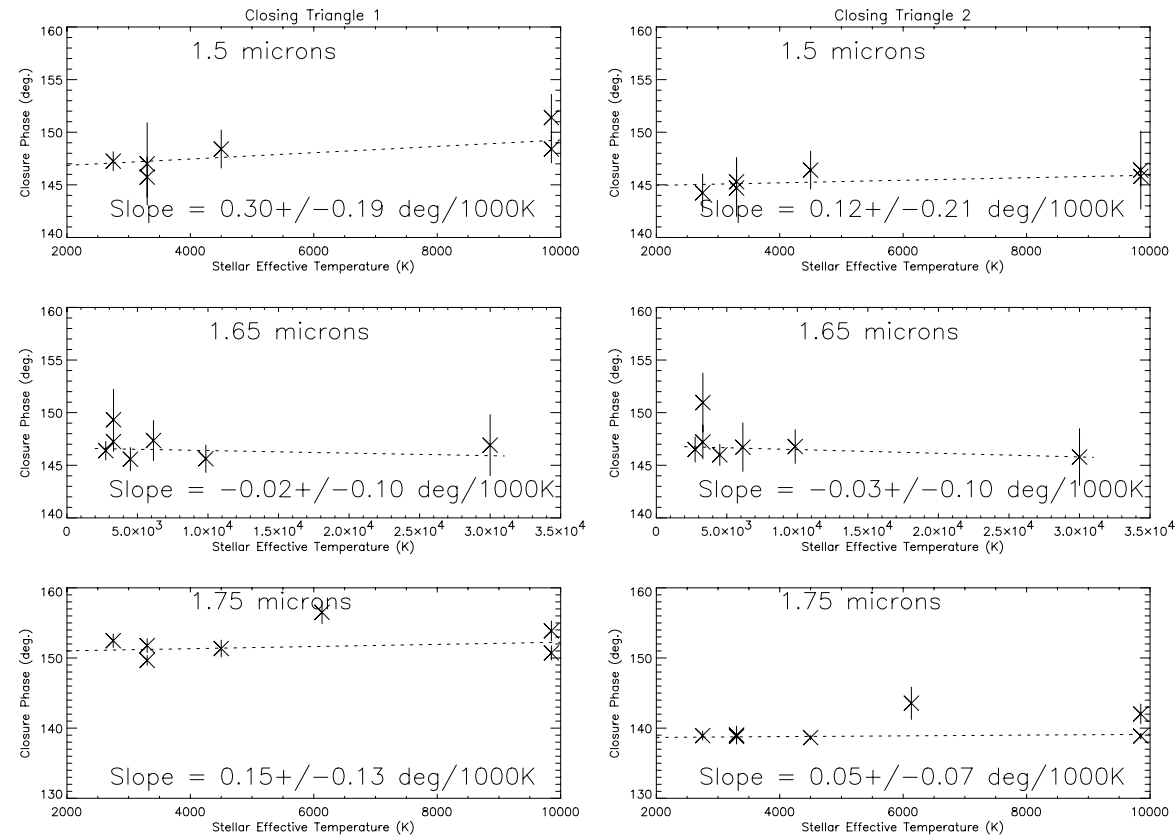

Figure 6. Narrowband closure phase measurements of calibrators observed during AGB run in March 2003 are plotted against stellar effective temperature. The left column corresponds to PICNIC pixels: $0,2 \& 4$ (closing triangle 1) and the right column corresponds to complementary outputs (closing triangle 2). 


\subsection{Polarization effects of the IOTA-IONIC}

The effect of polarization on visibility amplitude and closure phase measurements are studied by introducing a polarizer after the beam-combiner at an appropriate orientation in order to select one polarization component at a time. These results are shown in Figure 7. The instrumental fringe contrast improves when only one polarization component is selected. However, there is no significant change in the instrumental closure between these two cases, in general the maximum difference in the closure phase between these two cases is about six degrees.
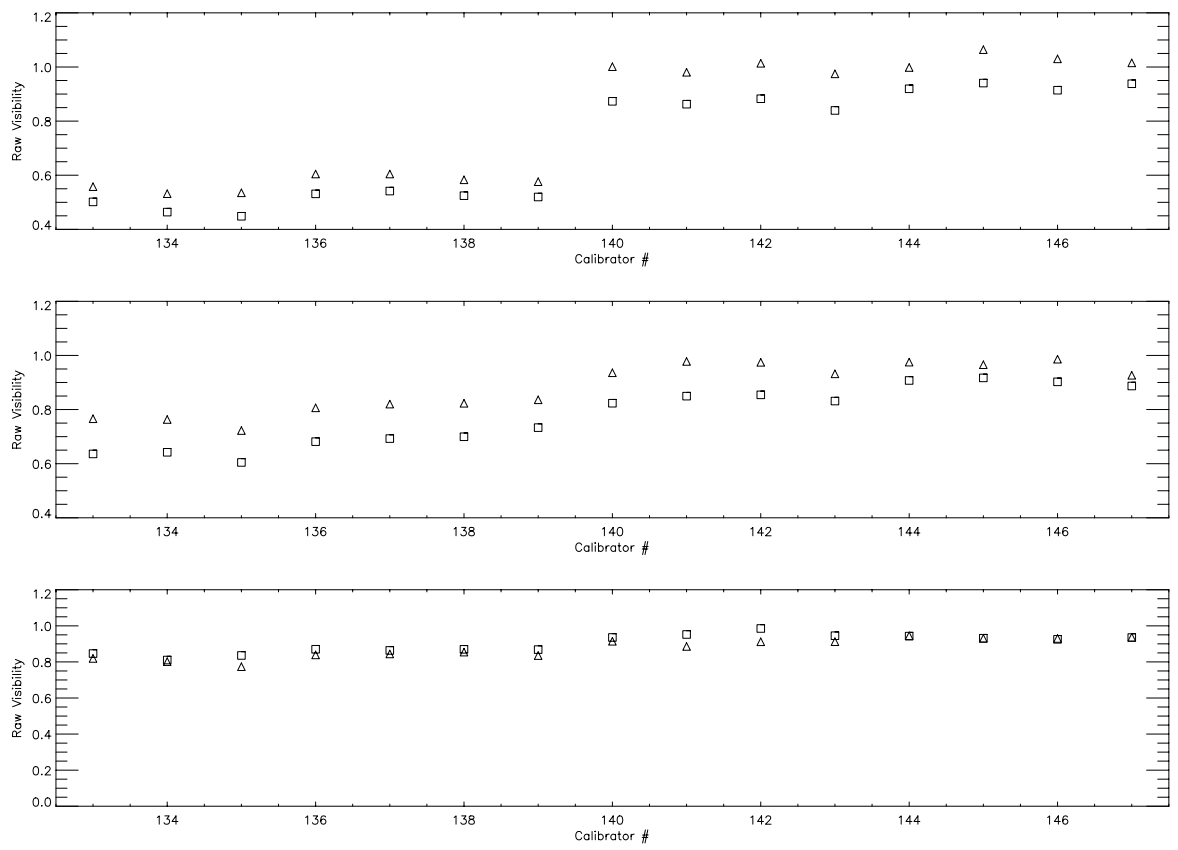

Figure 7. Measured visibility amplitude as a function of observation number are shown for all three baselines. A jump seen at observation number 140 corresponds to the time when a polarizer is introduced in order to select only one polarization component.

\subsection{Instrumental closure phase as a function of PICNIC readout pattern}

The dependence of the PICNIC readout pattern on the instrumental closure phase is shown in Figure 8 . The dependence is stronger for number of loops and weaker for number of reads.

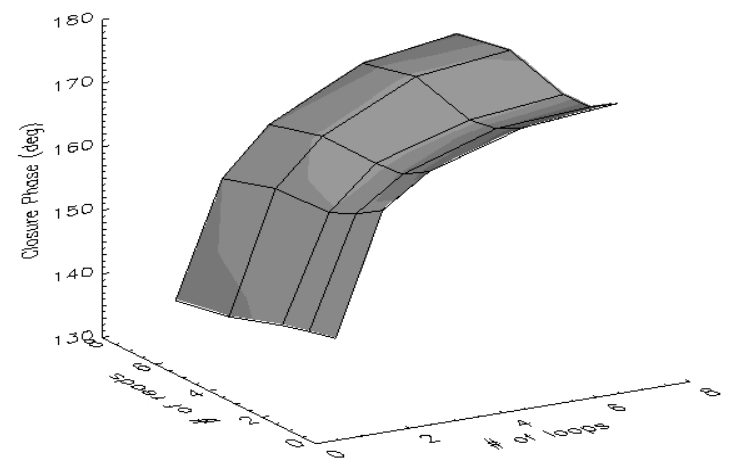

Figure 8. The loop and read dependence of the instrumental closure phase is shown in this plot. 


\subsection{Sensitivity of the Instrument}

The flux levels of the PICNIC camera at the IOTA are characterized in the H band from data taken on 07 Dec. 2003. Four calibrators of spectral type between early B type and late $\mathrm{K}$ type are used. The readout mode adopted for these observations is 1 Loop and 4 Reads. Figure 9 shows observed PICNIC outputs as a function of $\mathrm{H}$ magnitude for 1 Loop and 4 Reads. While measuring flux from a given telescope beam, the other two beams are blocked. The background light levels are also taken into account. The values plotted here are the average values of the four pixels illuminated by each telescope. The Zero H-magnitude flux is 925 ADU for 1 loop and 4 reads.

Since $\mathrm{K}$ magnitudes are more easily available in the literature than $\mathrm{H}$ magnitude, the expected picnic outputs are also calibrated as a function of $\mathrm{K}$ magnitudes. The Zero K-magnitude flux is 838 ADU for 1 loop and 4 reads.

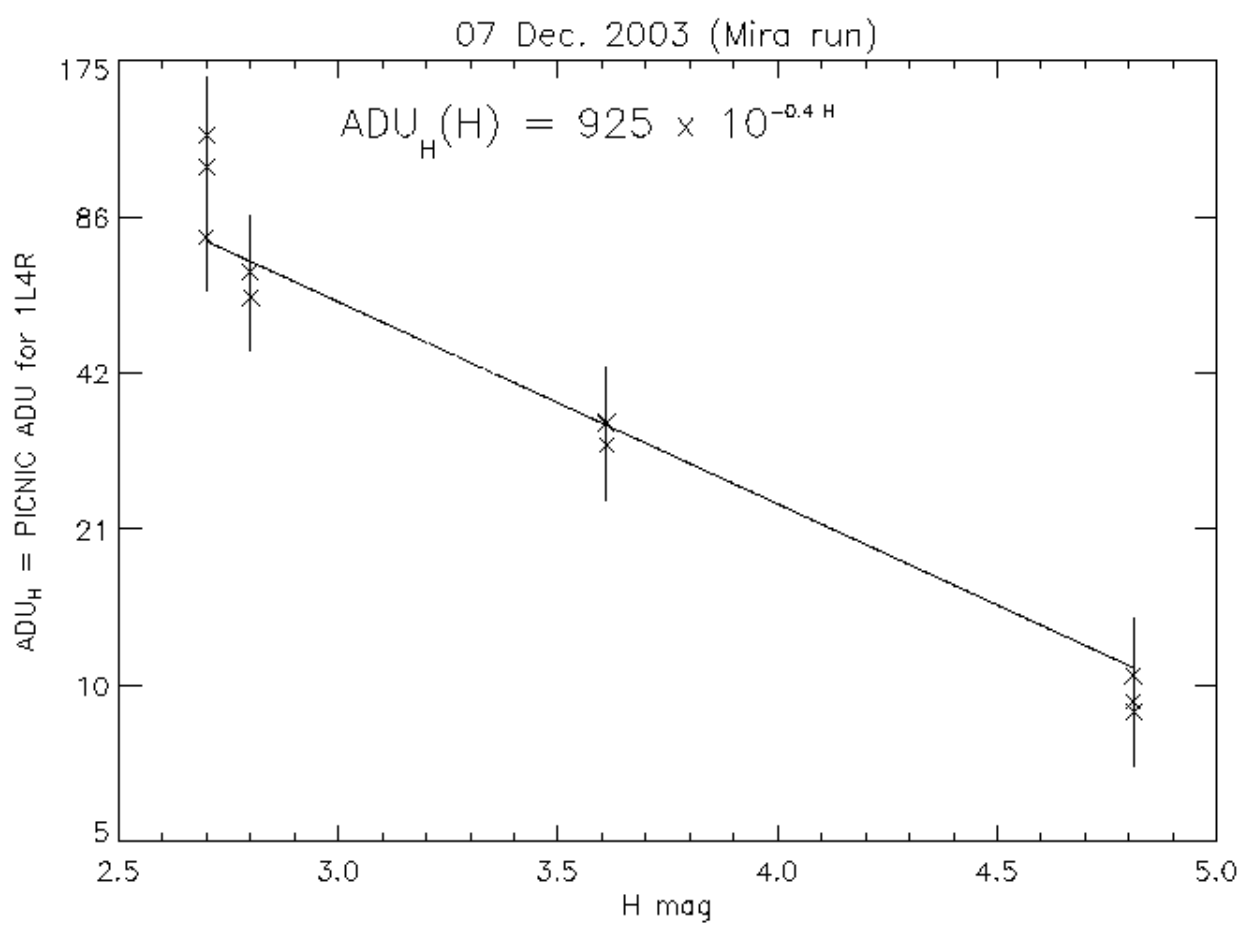

Figure 9. The picnic outputs (ADU) in $\mathrm{H}$ band, for the readout mode of 1 Loop and 4 Reads, are plotted against $\mathrm{H}$ magnitudes. A straight line fit with a slope of -0.4 is also shown here.

The present faint limit with the IONIC beam-combiner is $\mathrm{H} \sim 7$ for the broad band and $\mathrm{H} \sim 5$ for the three narrow band filters. For the difficult case of observing well resolved Mira stars with the $\mathrm{H}$ filter at or below $5 \%$ visibility level, the limiting magnitude is $\mathrm{H} \sim 4$. The limiting magnitude of the existing star tracker system at the IOTA is $\mathrm{V} \sim 12$ for latetype stars.

\section{ACKNOWLEDGEMENT}

This work was performed in part under contract with the Jet Propulsion Laboratory (JPL) through the Michelson Fellowship to Ragland, funded by NASA as an element of the Navigator (planet finder) program. JPL is managed for NASA by the California Institute of Technology. 


\section{REFERENCES}

Berger, J.-P., Haguenauer, P., Kern, P., Rousselet-Perraut, K., Malbet, F. et al., 2003, “An integrated-optics 3-way beam combiner for IOTA" in Interferometry for Optical Astronomy II, Traub, W.A., Ed., Proc. SPIE 4838, 1099

Pedretti, E. et al., "Fringe Tracking at the IOTA interferometer", in Proc. SPIE, New Frontiers in Stellar Interferometer, Wesley A. Traub; Ed., 5491, 2004

Ragland, S. et al., "First Surface-resolved results with the IOTA Imaging Interferometer: Detection of Asymmetric Structures in AGB stars" ApJ (lett), 2004 (submitted)

Traub, W.A., Berger, J.-P., Brewer, M.K., Carleton, N.P., Kern, P. et al., "IOTA: Recent Technology and Science", in Proc. SPIE, New Frontiers in Stellar Interferometer, Wesley A. Traub; Ed., 5491, 2004 